\title{
OPEN Prognostic value of thrombin generation parameters in hospitalized COVID-19 patients
}

\author{
María Eugenia de la Morena-Barrio ${ }^{1,4}$, Carlos Bravo-Pérez ${ }^{1,4}$, Antonia Miñano ${ }^{1}$, \\ Belén de la Morena-Barrio ${ }^{1}$, María Piedad Fernandez-Perez ${ }^{1}$, Enrique Bernal ${ }^{2}$, \\ José Miguel Gómez-Verdu ${ }^{3}$, María Teresa Herranz ${ }^{3}$, Vicente Vicente ${ }^{1}$, Javier Corral ${ }^{1 \bowtie}$ \& \\ María Luisa Lozano ${ }^{1}$
}

SARS-CoV-2 infection increases the risk of thrombosis by different mechanisms not fully characterized. Although still debated, an increase in D-dimer has been proposed as a first-line hemostasis test associated with thromboembolic risk and unfavorable prognosis. We aim to systematically and comprehensively evaluate the association between thrombin generation parameters and the inflammatory and hypercoagulable state, as well as their prognostic value in COVID-19 patients. A total of 127 hospitalized patients with confirmed COVID-19, 24 hospitalized patients with SARS-CoV-2-negative pneumonia and 12 healthy subjects were included. Clinical characteristics, thrombin generation triggered by tissue factor with and without soluble thrombomodulin, and also by silica, as well as other biochemical parameters were assessed. Despite the frequent use of heparin, COVID-19 patients had similar thrombin generation to healthy controls. In COVID-19 patients, the thrombin generation lag-time positively correlated with markers of cell lysis (LDH), inflammation (CRP, IL-6) and coagulation (D-dimer), while the endogenous thrombin potential (ETP) inversely correlated with D-dimer and LDH, and positively correlated with fibrinogen levels. Patients with more prolonged lag-time and decreased ETP had higher peak ISTH-DIC scores, and had more severe disease (vascular events and death). The ROC curve and Kaplan Meier estimate indicated that the D-dimer/ETP ratio was associated with in-hospital mortality (HR 2.5; $p=0.006)$, and with the occurrence of major adverse events (composite end-point of vascular events and death) (HR 2.38; $p=0.004$ ). The thrombin generation ETP and lag-time variables correlate with thromboinflammatory markers, and the D-dimer/ETP ratio can predict major adverse events in COVID-19.

The clinical manifestations of SARS-CoV-2 infection are highly heterogeneous, from asymptomatic disease to devastating outcomes. The most characteristic complication is acute respiratory distress syndrome (ARDS), but thrombotic complications are also frequent, especially in patients admitted to the intensive care unit (ICU) $)^{1-8}$. Thrombosis may be justified by excessive inflammation, hypoxia, immobilization, and microvascular injury ${ }^{9-14}$. Despite the strides that have been made in the last months in understanding the mechanisms underlying COVID19 associated coagulopathy, efforts are still necessary to decipher its complexity. The hemostatic profile of COVID-19 infection is different from sepsis coagulopathy or disseminated intravascular coagulation (DIC) ${ }^{15-20}$. Endothelial dysfunction and platelet activation may support thrombin generation. The activation of the complement system pathways may contribute to the production of cytokines and chemokines, and also phenomena of hypofibrinolysis could ultimately lead to immunothrombosis ${ }^{21}$. With limited solid data available, it is crucial to identify biomarkers that may help predict poor outcomes, including thrombotic events. In addition to D-dimer, most attempts have been mainly focused on the detection of new thromboinflammatory biomarkers, but studies analyzing global hemostatic methods are rare. In this disease, the correlation of thrombin generation parameters with biomarkers of immunothrombosis or with clinical outcomes has not been evaluated, which could provide some additional useful information not only in our understanding of the physiopathology of the disease but could also help for stratification and managed care of COVID-19. The aim of this study was to systematically

\footnotetext{
${ }^{1}$ Servicio de Hematología y Oncología Médica, Hospital General Universitario Morales Meseguer, Centro Regional de Hemodonación, Universidad de Murcia, IMIB-Arrixaca, CIBERER, Avda. Marqués de Los Vélez, s/n, 30008 Murcia, Spain. ${ }^{2}$ Servicio de Enfermedades Infecciosas, Hospital Reina Sofía, Murcia, Spain. ${ }^{3}$ Servicio de Medicina Interna, Hospital Universitario Morales Meseguer, Murcia, Spain. ${ }^{4}$ These authors contributed equally: María Eugenia de la Morena-Barrio and Carlos Bravo-Pérez email: Javier.corral@carm.es
} 
investigate the potential association of thrombin generation with other biochemical variables, and to assess the prognostic value of this assay in patients with COVID-19.

\section{Materials and methods}

Patients. A total of 142 consecutive patients with COVID-19 requiring hospital admission at Morales Meseguer and Reina Sofia University Hospitals (Murcia, Spain) from March to April 2020 were eligible for this cross-sectional study. In all patients, SARS-CoV-2 infection was assessed by quantitative real-time reversetranscriptase polymerase chain reaction (qRT-PCR) using the diagnostic criteria defined in the World Health Organization (WHO) interim guidance ${ }^{22}$. Exclusion criteria included chronic oral anticoagulation at the time of blood sampling, patients younger than 18 years of age, or those with missing data or who were transferred from other designated hospitals. Final follow-up date July 14, 2020.

As comparative groups, patients requiring admission at the same period with SARS-CoV-2-negative pneumonia were also enrolled. In these cases, COVID-19 diagnosis was ruled out by repetitively negative SARS-CoV-2 qRT-PCR and serologic testing. Samples from 12 healthy individuals and a pool of plasma from 100 healthy subjects were also analyzed for thrombin generation.

Ethics approval and consent to participate. All included subjects gave their informed consent to enter the study, which was approved by the Ethics Committee of Morales Meseguer Hospital, and performed in accordance with the 1964 Declaration of Helsinki and their later amendments.

Sample collection for thrombin generation analysis. Samples were collected during hospitalization in vacuum tubes containing $0.109 \mathrm{M}$ sodium citrate. Platelet-poor plasma (PPP) was obtained within $4 \mathrm{~h}$ after extraction by two cycles of centrifugation $(2000 \mathrm{~g} \times 15 \mathrm{~min})$ at $20^{\circ} \mathrm{C}$ and then stored at $-80^{\circ} \mathrm{C}$.

Data collection. Three investigators (MTH, JMGV, and EB) independently extracted data including demographics, comorbidities, laboratory studies and outcomes, using a standardized data collection form for analysis. Such data were checked independently by three investigators (CBP, MPFP and MEMB) to verify accuracy. The clinical information comprised patient demographics, medical comorbidities, prior thrombotic history, chronic anticoagulation, length of hospital stay, CURB-65 score at admission, development of ARDS, ICU admission, sequential organ failure assessment (SOFA) score, vascular (thrombotic/ hemorrhagic) events, antithrombotic therapy while hospitalization, and death.

Venous and arterial thrombotic events were confirmed by radiologic methods (Doppler-Ultrasonography [US] and Computed Tomography [CT]). Bleeding was graded according to World Health Organization (WHO) scale. Grade $\geq 3$ bleeding episodes, requiring transfusion, were confirmed by angiographic procedures. Routine laboratory parameters: lactate dehydrogenase (LDH), C reactive protein (CRP), interleukin 6 (IL-6), complete blood cell counts, basic coagulation tests (prothrombin time [PT], activated partial thromboplastin time [aPTT], fibrinogen and D-dimer), were recorded at admission. They were then assessed at average intervals of $48 \mathrm{~h}$, and their peak levels during follow-up were also documented. COVID-19 associated coagulopathy was graded according to DIC score of the International Society on Thrombosis and Haemostasis (ISTH) ${ }^{23}$. Admission and peak ISTH-DIC scores were calculated. Prescribed antithrombotic therapy with low molecular weight heparin (LMWH), specifically enoxaparin, was classified as standard prophylaxis (40 mg QD), high-risk prophylaxis ( $1 \mathrm{mg} / \mathrm{Kg} \mathrm{QD})$, and therapeutic regimens $(1 \mathrm{mg} / \mathrm{Kg} \mathrm{BD})$.

Thrombin generation assay. The thrombin generation assay (TGA) was performed using the calibrated automated thrombogram (CAT) method ${ }^{24}$. All samples were evaluated in the same equipment during three working days using the same reagent batch under the following conditions: samples were thawed at $37^{\circ} \mathrm{C}$ and mixed with two different activators: PPP reagent (5 pM tissue factor [TF]; $4 \mu \mathrm{M}$ phospholipids) (Stago Diagnostics) or silica (dilution 1/16 from Synthasyl reagent; Werfen), in a 96-well plate (Immulon, $2 \mathrm{HB}$ clear U-bottom; ThermoFisher Scientific). All samples were run in duplicate. Coagulation was triggered with calcium chloride in buffer containing the fluorogenic substrate (FluCa-kit reagent, Stago Diagnostics). For each individual plasma sample, we used a thrombin calibrator (Stago Diagnostics) to correct for differences in sample color, inner filter fluorescence, and substrate consumption. Fluorescence was recorded for $60 \mathrm{~min}$ in a Fluoroskan Ascent microplate fluorimeter (Thermolab Systems, Helsinki, Finland), and the data were then analyzed using Thrombinoscope software (version 5.0.0.742, Stago Diagnostics). The endogenous thrombin potential (ETP), the thrombin peak, the time to the thrombin peak, the velocity index and the lag-time were recorded for each assay. To assess the impact of the protein $\mathrm{C}$ anticoagulant pathway on the TGA, experiments activated with PPP reagent were also conducted in the presence of recombinant human soluble thrombomodulin (sTM) (American Diagnostica) at a final concentration of $7.5 \mathrm{nM}$. An index " $\mathrm{R}$ " described by Perrin et $\mathrm{al}^{25}$, was calculated for the TGA parameters, i.e. the value in the presence of sTM divided by the value in the absence of sTM; the closer the ratio is to 1 , the weaker the response to the protein $\mathrm{C}$ system.

This assay was done in the baseline sample for both COVID-19 and the non-COVID-19 pneumonia groups.

Statistical analysis. Descriptive analysis of qualitative variables included percentages. Pearson's ChiSquared test and Fisher's Exact test were used for comparison of proportions or ordinal variables. KolmogorovSmirnov and Shapiro-Wilk tests were used for testing normality of continuous variables. Normally distributed continuous variables were presented as means \pm standard deviations (SD), whereas non-normally distributed variables were presented as median and interquartile ranges (IQR). Student's-T (parametric) or Mann-Whitney- 


\begin{tabular}{|c|c|c|c|}
\hline & COVID-19 $(\mathrm{N}=127)$ & SARS-CoV-2-negative Pneumonia $(\mathrm{N}=24)$ & $p$ \\
\hline \multicolumn{4}{|l|}{ Demographics } \\
\hline Sex (females) & $43.3 \%(\mathrm{~N}=55)$ & $54.2 \%(\mathrm{~N}=13)$ & 0.327 \\
\hline Age (years), median (IQR) & $60(47.5-72.3)$ & $37(29.5-31.8)$ & $<0.001$ \\
\hline \multicolumn{4}{|l|}{ Comorbidities } \\
\hline Hypertension & $47.0 \%(\mathrm{~N}=61)$ & $12.5 \%(\mathrm{~N}=3)$ & 0.002 \\
\hline Diabetes mellitus & $18.1 \%(\mathrm{~N}=23)$ & $0 \%$ & 0.016 \\
\hline Dyslipidemia & $36.2 \%(\mathrm{~N}=46)$ & $0 \%$ & $<0.001$ \\
\hline Cardiovascular disease & $14.2 \%(\mathrm{~N}=18)$ & $4.2 \%(\mathrm{~N}=1)$ & 0.135 \\
\hline Chronic lung disease & $1.6 \%(\mathrm{~N}=2)$ & $8.3 \%(\mathrm{~N}=2)$ & 0.089 \\
\hline Smoking history & $17.3 \%(\mathrm{~N}=22)$ & $37.5 \%(\mathrm{~N}=9)$ & 0.049 \\
\hline Chronic renal disease & $18.9 \%(\mathrm{~N}=24)$ & $0 \%$ & 0.020 \\
\hline Cancer & $4.7 \%(\mathrm{~N}=6)$ & $0 \%$ & 0.590 \\
\hline Immunosuppression & $4.7 \%(\mathrm{~N}=6)$ & $0 \%$ & 0.590 \\
\hline \multicolumn{4}{|l|}{ At-admission severity } \\
\hline CURB-65 $\geq 2, \%(95 \% \mathrm{CI})$ & $30.7 \%(22.8-39.5 \%)(\mathrm{N}=39)$ & $0 \%(0-14.2 \%)$ & 0.002 \\
\hline Length of stay, median (IQR) & 10 days ( $5-19$ days) & 2 days (1-3.8 days) & $<0.001$ \\
\hline \multicolumn{4}{|l|}{ Evolution to critically-ill disease } \\
\hline ARDS, \% (95\%CI) & $30.7 \%(22.8-39.5 \%)(\mathrm{N}=33)$ & $0 \%(0-14.2 \%)$ & 0.002 \\
\hline ICU admission, \% (95\%CI) & $26.0 \%(18.6-34.5 \%)(\mathrm{N}=39)$ & $0 \%(0-14.2 \%)$ & 0.005 \\
\hline SOFA score, median (IQR) & $0(0-2)$ & $0(0-1)$ & 0.020 \\
\hline SOFA of ICU patients, median (IQR) & $4(0-7.5)$ & - & - \\
\hline \multicolumn{4}{|l|}{ Deaths, \% (95\%CI) } \\
\hline Overall & $7.9 \%(3.8-14.0 \%)(\mathrm{N}=10)$ & $0 \%$ & $0.004^{\star}$ \\
\hline ICU & $18.2 \%(7.0-35.5 \%)(\mathrm{N}=6)$ & $(0-14.2 \%)$ & \\
\hline Non-ICU & $4.3 \%(1.3-4.2 \%)(\mathrm{N}=4)$ & & \\
\hline
\end{tabular}

Table 1. Demographic and clinical characteristics of patients with COVID-19 and SARS-CoV-2 negative pneumonia. Bold values denote statistical significance at the $p<0.05$ level. ARDS acute respiratory distress syndrome, ICU intensive care unit, IQR interquartile range, SOFA score Sequential organ failure assessment score. ${ }^{\star} p$ value of Chi-Squared test for 3 categories (COVID-19 ICU and non-ICU and SARS-CoV-2-negative Pneumonia) and $p$ value of 0.019 for deaths in ICU $v s$. non-ICU COVID-19 patients.

U tests were used for comparison of two means and analysis of variance (ANOVA, parametric) or Kruskal-Wallis (non-parametric) tests were used for comparison of more than two means. Apart from $p$ values, 95\% Confidence Intervals $(95 \% \mathrm{CI})$ were also calculated.

Association between thrombin generation and other continuous laboratory variables was assessed by Pearson's (parametric) or Spearman's Rho (non-parametric) correlation tests.

The predictive impact of thrombin generation and other laboratory parameters on the incidence of clinical events (hemorrhage, thrombosis, ARDS, ICU admission and death) was assessed by Cox regression analysis. Survival analysis was assessed using the Kaplan-Meier method and log-rank tests. Receiver-Operating-Characteristic (ROC) curve analysis was also performed, and optimal cut-off values were selected by means of maximizing sensitivity and specificity. Logarithmic and square root transformations of continuous variables were performed to make the data more closely meet the assumptions of statistical procedures to be applied, or to improve their interpretability.

Statistical analysis was performed with the use of Excel (Microsoft), GraphPad Prism (GraphPad Software), IBM SPSS Statistics 21 (IBM SPSS Software) and STATA v.14 (StataCorp LLC) ${ }^{26}$.

\section{Results}

Patients' characteristics. A total of 127 out of 142 hospitalized COVID-19 patients were eventually included in the study. Fifteen patients were excluded: six due to oral anticoagulation uptake at the time of blood sampling and nine because of missing data or transfer from other hospitals.

The basic characteristics for all included participants are shown in Table 1. COVID-19 patients were older than those with SARS-CoV-2 negative pneumonia (median age 60 years [IQR, 47.5-72.3 years] vs. 37 years [IQR 29.5-31.7 years]), respectively; $p<0.001$ ). The median time from admission to discharge was 10 days in COVID-19 patients, and 2 days in SARS-CoV-2 negative pneumonia patients $(p=0.002)$. COVID-19 patients had significantly higher rates of hypertension, diabetes, dyslipidemia, and chronic renal failure, whereas SARSCoV-2 pneumonia patients had higher rates of history of smoking $(p \leq 0.05)$. The results also showed that $30.7 \%$, and $26.0 \%$ of COVID-19 patients developed ARDS and required ICU admission, respectively, and that $7.9 \%$ of the COVID-19 cohort had fatal outcomes. Patients in the SARS-CoV-2-negative pneumonia group had much milder clinical manifestations. 


\begin{tabular}{|l|l|l|l|}
\hline \multicolumn{2}{|l|}{ COVID-19 (N=127) } & SARS-CoV-2 negative Pneumonia (N=24) & $p$ \\
\hline Antithrombotic therapy & $22.0 \%(\mathrm{~N}=28)$ & $45.8 \%(\mathrm{~N}=11)$ & $\mathbf{0 . 0 1 5}$ \\
\hline None, \% & $65.4 \%(\mathrm{~N}=83)$ & $54.2 \%(\mathrm{~N}=13)$ & 0.296 \\
\hline Prophylactic LMWH, \% & $12.6 \%(\mathrm{~N}=16)$ & $0 \%$ & 0.076 \\
\hline Intermediate/treatment dose LMWH, \% & \multicolumn{2}{|l|}{} \\
\hline Thrombotic events, \% (95\%CI) & $5.5 \%(2.2-11.0 \%)(\mathrm{N}=7)$ & $0 \%(0-14.2 \%)$ & $\mathbf{0 . 0 0 4}^{\star}$ \\
\hline Overall & $15.2 \%(5.1-35.9 \%)(\mathrm{N}=5)$ & & \\
\hline ICU & $2.1 \%(0.3 \%-7.5 \%)(\mathrm{N}=2)$ & & $0.448^{\star}$ \\
\hline Non-ICU & $3.8 \%(1.2-8.6 \%)(\mathrm{N}=5)$ & $0 \%(0-14.2 \%)$ & \\
\hline Bleeding events, \% (95\%CI) & $6.1 \%(0.7-20.2 \%)(\mathrm{N}=2)$ & & \\
\hline Overall & $3.2 \%(0.7-9.0 \%)(\mathrm{N}=3)$ & & \\
\hline ICU & & & \\
\hline Non-ICU &
\end{tabular}

Table 2. Antithrombotic therapies and vascular events in COVID-19 patients and with SARS-CoV-2 negative pneumonia. Bold values denote statistical significance at the $p<0.05$ level. ICU intensive care unit, $L M W H$ low molecular weight heparin. ${ }^{*} p$ value of 0.013 for thrombotic events in ICU $v s$. non-ICU COVID-19 patients, and $p$ value of 0.604 for bleeding events in ICU $v s$. non-ICU COVID-19 patients.

Antithrombotic prophylaxis, thrombosis, and bleeding events in COVID-19 patients. Antithrombotic therapies, thrombotic events, and bleeding complications in patients with COVID-19 and with SARS-CoV-2 negative pneumonia are summarized in Table 2.

In the COVID-19 cohort, 7 patients experienced 8 thrombotic events. These included 5 venous episodes $(4$ pulmonary embolism [PE], 1 extensive superficial venous thrombosis), and 3 ischemic episodes (1 stroke, 1 acute coronary syndrome, and 1 acute peripheral arterial ischemia). Thus, the incidence of thrombosis in COVID-19 patients in our cohort was 5.5\% (95\%CI $2.2-11.0 \%) ; 3.9 \%$ (95\%CI 1.3-8.9\%) for radiographically-confirmed venous thromboembolism and $2.4 \%$ (95\%CI $0.5-6.7 \%)$ for arterial thrombosis. Five out of these 7 patients (71.4\%) had critically-ill COVID-19 and required ICU admission, rendering an incidence of thrombosis of $15.2 \%$ (95\% CI 5.1-31.9\%) in ICU patients. One of the patients developed two vascular events, a symptomatic PE followed 7 days later by acute myocardial infarction despite full-dose anticoagulation with heparin. Of note, thrombotic complications seemed to occur in spite of antithrombotic measures, since $85.8 \%$ of patients with thrombosis received pharmacological thromboprophylaxis, and $28.6 \%$ of them intermediate/therapeutic dose heparin.

Five bleeding events occurred in 5 COVID-19 patients: 2 retroperitoneal hematomas, 1 rectus sheath hematoma (these 3 episodes had a WHO Severity Grade $\geq 3$ ), 1 upper gastrointestinal bleeding and 1 episode of hemoptysis. Therefore, the rate of bleeding in COVID-19 patients from our cohort was 3.8\% (95\%CI 1.2-8.6\%) and $2.3 \%$ (95\%CI $0.5-6.5 \%)$ for WHO grade $\geq 3$ bleeding. Two out of these 5 patients $(40.0 \%)$ had critically-ill COVID-19 and required ICU admission, resulting in an incidence of bleeding of $6.1 \%(95 \% \mathrm{CI} 0.7-20.2 \%)$ in ICU patients. All cases received thromboprophylaxis with heparin. Notably, at the moment of bleeding, the 3 patients with Grade $\geq 3$ internal hemorrhages were on intermediate/therapeutic-dose heparin.

No vascular events were recorded in patients with SARS-CoV-2-negative pneumonia.

Laboratory data. For all patients, the baseline timepoint corresponded with the sample collected at a median of 2.0 days following admission (IQR: 1.0-7.0).

Baseline and peak levels of LDH and D-dimer were higher in COVID-19 patients than those in the SARSCoV-2 negative pneumonia group. In terms of blood counts and other coagulation indexes, the COVID-19 group showed significantly lower trough platelet counts and PT compared with the SARS-CoV-2 negative pneumonia group. Thus, in the COVID-19 cohort, $11.0 \%$ of patients had thrombocytopenia $\left(<150 \times 10^{9} / \mathrm{L}\right), 30.7 \%$ had prolonged PT $(<75 \%)$ and/or aPTT ratio $(>1.30)$ and $0.8 \%$ fibrinogen consumption $(<100 \mathrm{mg} / \mathrm{dL})$. Meanwhile, baseline and peak ISTH-DIC score values were also significantly higher in COVID-19 patients than those in the SARS-CoV-2 negative pneumonia group (Table 3).

Notably, at admission, no patient met criteria of overt DIC (score $\geq 5$ ), but in $51.9 \%$ of them, the profile was consistent with low-grade coagulopathy (scores 1-4). During follow-up, 64.7\% cases showed low-grade coagulopathy, and 3.8\% eventually met criteria of overt DIC. Both baseline and peak ISTH-DIC scores were higher in patients suffering from bleeding than those who did not (median baseline ISTH-DIC score: 2 [IQR: 1.25-2.75] for bleeders and 0 [IQR: 0-1] for non-bleeders, $p=0.005$; median peak ISTH-DIC score: 3.5 [IQR: 1.5-4] for bleeders and 1 [IQR: $0-2]$ for non-bleeders, $p=0.016$ ).

Thrombin generation. The results of the thrombin generation parameters in COVID-19 and SARS-CoV-2 negative pneumonia evaluated in baseline samples, as well as the reference values of healthy subjects are shown in Table 4. Although 78.9\% of COVID-19 patients $(\mathrm{N}=105)$ were undergoing LMWH treatment, when thrombin generation was triggered either by TF or silica, COVID-19 patients had similar lag-time and ETP but higher peak than healthy controls. At $5 \mathrm{pM}$ TF, SARS-CoV-2 negative pneumonia patients presented with a shorter 


\begin{tabular}{|c|c|c|c|c|c|}
\hline & \multicolumn{2}{|l|}{ Baseline } & \multicolumn{2}{|l|}{ Peak value $^{a}$} & \multirow[b]{2}{*}{ Normal range } \\
\hline & COVID-19 & $\begin{array}{l}\text { SARS-CoV-2-negative } \\
\text { Pneumonia }\end{array}$ & COVID-19 & $\begin{array}{l}\text { SARS-CoV-2-negative } \\
\text { Pneumonia }\end{array}$ & \\
\hline \multicolumn{6}{|c|}{ Inflammation markers } \\
\hline LDH, IU/L & $505 \pm 213$ & $336 \pm 65$ & $668 \pm 385$ & $381 \pm 77$ & $208-378$ \\
\hline$p$ & \multicolumn{2}{|l|}{$<0.001$} & \multicolumn{2}{|l|}{$<0.001$} & \\
\hline $\mathrm{CRP}, \mathrm{mg} / \mathrm{dL}$ & $8.2 \pm 8.3$ & $7.2 \pm 6.4$ & $11.1 \pm 9.8$ & $7.5 \pm 6.9$ & $0-1.0$ \\
\hline$p$ & \multicolumn{2}{|l|}{0.79} & \multicolumn{2}{|l|}{0.12} & \\
\hline IL-6, pg/mL & ND & $\mathrm{ND}$ & $98.6 \pm 304.7$ & $16.0 \pm 13.0$ & $0-4.4$ \\
\hline$p$ & \multicolumn{2}{|l|}{-} & \multicolumn{2}{|l|}{0.065} & \\
\hline \multicolumn{6}{|c|}{ Routine hemostatic tests } \\
\hline Platelets, $\times 10^{9} / \mathrm{L}$ & $216.2 \pm 87.3$ & $242.2 \pm 89.0$ & $194.9 \pm 87.3$ & $245.9 \pm 89.1$ & $150.0-450.0$ \\
\hline$p$ & \multicolumn{2}{|l|}{0.11} & \multicolumn{2}{|l|}{0.008} & \\
\hline PT, \% & $91.8 \pm 17.9$ & $93.6 \pm 12.6$ & $79.8 \pm 17.2$ & $85.0 \pm 13.3$ & $75-100$ \\
\hline$p$ & \multicolumn{2}{|l|}{0.68} & \multicolumn{2}{|l|}{0.22} & \\
\hline aPTT, ratio & $1.06 \pm 0.19$ & $1.04 \pm 0.12$ & ND & ND & $0.80-1.30$ \\
\hline$p$ & \multicolumn{2}{|l|}{0.79} & \multicolumn{2}{|l|}{-} & \\
\hline Fibrinogen, $\mathrm{mg} / \mathrm{dL}$ & $457 \pm 189$ & $482 \pm 150$ & $514 \pm 180$ & $508 \pm 153$ & $150.0-400.0$ \\
\hline$p$ & \multicolumn{2}{|l|}{0.76} & \multicolumn{2}{|l|}{0.74} & \\
\hline D-dimer, mg/dL & $1,308 \pm 2,042$ & $556 \pm 464$ & $4,162 \pm 9,492$ & $690 \pm 489$ & $0-500$ \\
\hline$p$ & \multicolumn{2}{|l|}{0.001} & \multicolumn{2}{|l|}{$<0.001$} & \\
\hline \multicolumn{6}{|l|}{ ISTH-DIC score } \\
\hline Median, IQR & $0(0-1)$ & $0(0-0.25)$ & $1(0-2)$ & $0(0-1)$ & $<1$ \\
\hline$p$ & \multicolumn{2}{|l|}{0.024} & \multicolumn{2}{|l|}{0.001} & \\
\hline
\end{tabular}

Table 3. Baseline and peak laboratory parameters in COVID-19 patients and in those with SARS-CoV-2 negative pneumonia. Data are shown as mean \pm SD. Bold values denote statistical significance at the $p<0.05$ level. aPTT activated partial thromboplastin time, CRP C-reactive protein, $I L-6$ interleukin-6, $L D H$ lactate

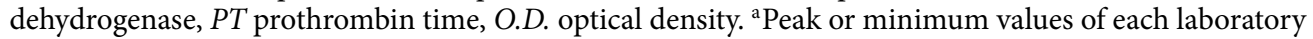
parameter are shown, depending on its upward or downward tendency during COVID-19, respectively. $P$ values were based on Mann-Whitney-U test or T-test.

\begin{tabular}{|c|c|c|c|c|c|c|}
\hline TGA parameter & Activator & COVID-19 $(\mathrm{N}=127)$ & $\begin{array}{l}\text { SARS-CoV-2 } \\
\text { negative }(N=24)\end{array}$ & $p$ & $\begin{array}{l}\text { Healthy subjects } \\
(\mathrm{N}=12) \& \text { pool }^{\star}\end{array}$ & $p$ (vs COVID-19) \\
\hline \multirow{2}{*}{ Lag-time (min) } & $\mathrm{TF}(5 \mathrm{pM})$ & $3.83[3.33-4.50]$ & $3.33[3.00-3.79]$ & 0.009 & $3.36[2.69-4.50]$ & 0.284 \\
\hline & Silica $(1 / 16)$ & $3.00[2.50-3.33]$ & $3.02[2.67-3.36]$ & 0.527 & $3.33[2.59-3.43]$ & 0.502 \\
\hline \multirow{2}{*}{ ETP (nM/min) } & $\mathrm{TF}(5 \mathrm{pM})$ & 1263 [1101-1488] & 1506 [1235-1747] & 0.005 & 1169 [1092-1732] & 0.376 \\
\hline & Silica $(1 / 16)$ & $1390[1205-1610]$ & 1556 [1304-1851] & 0.011 & $1501[1440-1732]$ & 0.144 \\
\hline \multirow{2}{*}{ Peak (nM) } & $\mathrm{TF}(5 \mathrm{pM})$ & 224 [179-256] & 237.3 [206-269] & 0.037 & $119[92-236]$ & 0.011 \\
\hline & Silica $(1 / 16)$ & 191 [166-222] & $190.0[172-225]$ & 0.696 & 171 [157-240] & 0.392 \\
\hline \multirow{2}{*}{ Tt Peak (min) } & $\mathrm{TF}(5 \mathrm{pM})$ & $6.67[6.00-7.67]$ & $6.20[5.33-6.86]$ & 0.030 & 8.54 [5.36-11.67] & 0.226 \\
\hline & Silica $(1 / 16)$ & $5.83[5.17-6.50]$ & $6.00[5.40-6.70]$ & 0.411 & $6.67[5.60-6.85]$ & 0.141 \\
\hline \multirow{2}{*}{ Vel Index (nM/min) } & $\mathrm{TF}(5 \mathrm{pM})$ & $79.3[56.6-103.3]$ & 91.8 [70.8-111.9] & 0.139 & $23.1[13.8-90.6]$ & 0.007 \\
\hline & Silica $(1 / 16)$ & $67.0[55.1-84.1]$ & $64.5[58.3-83.9]$ & 0.800 & $49.7[45.6-87.5]$ & 0.147 \\
\hline Ratio ETP sTM/No & $\mathrm{TF}(5 \mathrm{pM})$ & $0.86[0.76-0.91]$ & $0.86[0.82-0.89]$ & 0.706 & $0.63[0.52-0.89]$ & 0.023 \\
\hline
\end{tabular}

Table 4. Baseline thrombin generation parameters of plasma samples from pneumonia patients with or without COVID-19 and healthy controls. Values are expressed as median [IQR]. Bold values denote statistical significance at the $p<0.05$ level. ${ }^{\star}$ Plasma pool of 100 blood donors. $s T M$ soluble thrombomodulin, TF Tissue factor, TGA thrombin generation assay, Tt Peak time to peak, Vel Index velocity index. $P$ values were based on Mann-Whitney-U test.

lag-time, higher ETP and higher peak height compared to COVID-19 samples, most likely due to the lower proportion of patients receiving LMWH than COVID-19 patients (Table 4 and Fig. S1). In the study sample, when sTM was added, as an agent that converts thrombin into an anticoagulant capable of activating protein C, it could not significantly reduce the ETP in patients with pneumonia (COVID-19 and SARS-CoV-2 negative), compared with the effect expected and observed in samples from healthy subjects (Table 4).

Correlation of thrombin generation parameters with analytical data in COVID-19 patients. Analysis of the association between baseline TGA parameters and peak values of all other ana- 


\begin{tabular}{|c|c|c|c|c|c|c|c|c|c|c|}
\hline \multirow[b]{2}{*}{$\operatorname{Median}(\mathrm{N})$} & \multicolumn{2}{|l|}{ LDH (IU/L) } & \multicolumn{2}{|l|}{ CRP (mg/L) } & \multicolumn{2}{|c|}{ IL-6 (pg/mL) } & \multicolumn{2}{|c|}{ Fib (mg/dL) } & \multicolumn{2}{|l|}{$\mathrm{D}-\mathrm{D}(\mathrm{mg} / \mathrm{L})$} \\
\hline & $<564(62)$ & $>564(65)$ & $<8.6(71)$ & $>8.6(56)$ & $<13.8(75)$ & $>13.8(52)$ & $<446(66)$ & $>446(61)$ & $<1.24(63)$ & $>1.24(64)$ \\
\hline \multicolumn{11}{|l|}{ Lag-time (min) } \\
\hline Mean \pm SD & $3.7 \pm 1.1$ & $4.3 \pm 1.2$ & $3.9 \pm 1.3$ & $4.2 \pm 0.9$ & $3.8 \pm 1.1$ & $4.3 \pm 1.1$ & $4.1 \pm 1.3$ & $3.9 \pm 0.9$ & $3.7 \pm 0.7$ & $4.4 \pm 1.3$ \\
\hline$p^{*}$ & \multicolumn{2}{|l|}{0.022} & \multicolumn{2}{|l|}{0.01} & \multicolumn{2}{|l|}{0.004} & \multicolumn{2}{|l|}{0.969} & \multicolumn{2}{|l|}{$<0.001$} \\
\hline Correlation Rho & \multicolumn{2}{|l|}{0.247} & \multicolumn{2}{|l|}{0.0 .218397} & \multicolumn{2}{|l|}{0.407} & \multicolumn{2}{|l|}{0.068} & \\
\hline$p^{* *}$ & \multicolumn{2}{|l|}{0.007} & \multicolumn{2}{|l|}{$<0.0010 .021$} & \multicolumn{2}{|l|}{$<0.001$} & \multicolumn{2}{|l|}{0.461} & & \\
\hline \multicolumn{11}{|l|}{ ETP (nM/min) } \\
\hline Mean \pm SD & $1350 \pm 320$ & $1222 \pm 270$ & $1267 \pm 330$ & $1307 \pm 260$ & $1264 \pm 300$ & $1314 \pm 300$ & $1235 \pm 340$ & $1337 \pm 240$ & $1354 \pm 280$ & $1216 \pm 300$ \\
\hline$p^{*}$ & \multicolumn{2}{|l|}{0.016} & \multicolumn{2}{|l|}{0.19} & \multicolumn{2}{|l|}{0.368} & \multicolumn{2}{|l|}{0.022} & \multicolumn{2}{|l|}{0.019} \\
\hline Correlation Rho & \multicolumn{2}{|l|}{-0.241} & \multicolumn{2}{|l|}{0.024} & \multicolumn{2}{|l|}{-0.046} & \multicolumn{2}{|l|}{0.300} & \multicolumn{2}{|l|}{-0.288} \\
\hline$p^{* *}$ & \multicolumn{2}{|l|}{0.009} & \multicolumn{2}{|l|}{0.804} & \multicolumn{2}{|l|}{0.647} & \multicolumn{2}{|l|}{0.001} & \multicolumn{2}{|l|}{\begin{tabular}{|l|}
0.001 \\
\end{tabular}} \\
\hline
\end{tabular}

Table 5. Association between baseline TGA parameters (lag-time and ETP) induced by TF (5 pM) and peak analytical data in COVID-19 patients. Bold values denote statistical significance at the $p<0.05$ level. Peak values of LDH, CRP, IL-6, Fib and D-D are dichotomized according to the median. ${ }^{\star}$ Mann-Whitney-U test and on ${ }^{\star \star}$ Spearman's Rho correlations. Rho Spearman's Rho (range: -1.00 to +1.00 ), ETP Endogenous thrombin potential, $L D H$ Lactate dehydrogenase, CRP C reactive protein, $I L$ Interleukin, O.D. optical density, Fib Fibrinogen, D-D D-dimer.

\begin{tabular}{|c|c|c|c|c|c|c|c|c|c|c|c|c|}
\hline & \multicolumn{2}{|l|}{ CURB-65 } & \multicolumn{2}{|l|}{ ICU } & \multicolumn{2}{|l|}{ ARDS } & \multicolumn{2}{|l|}{ Death } & \multicolumn{2}{|c|}{ Thrombosis } & \multicolumn{2}{|c|}{ Complication ${ }^{\star}$} \\
\hline & $\begin{array}{l}0-1 \\
(\mathrm{~N}=88)\end{array}$ & $\geq 2(\mathrm{~N}=39)$ & No $(\mathrm{N}=94)$ & Yes $(\mathrm{N}=33)$ & No $(\mathrm{N}=88)$ & Yes $(\mathrm{N}=39)$ & $\begin{array}{l}\text { No } \\
(\mathrm{N}=117)\end{array}$ & $\begin{array}{l}\text { Yes } \\
(\mathrm{N}=10)\end{array}$ & $\begin{array}{l}\text { No } \\
(\mathrm{N}=120)\end{array}$ & Yes $(\mathrm{N}=7)$ & No $(\mathrm{N}=86)$ & Yes $(\mathrm{N}=41)$ \\
\hline Lag-time & $3.8 \pm 0.9$ & $4.5 \pm 1.4$ & $3.9 \pm 1.1$ & $4.4 \pm 1.2$ & $3.9 \pm 1.0$ & $4.4 \pm 1.3$ & $4.0 \pm 1.2$ & $4.1 \pm 0.9$ & $3.9 \pm 1.0$ & $5.8 \pm 2.3$ & $3.8 \pm 0.9$ & $4.5 \pm 1.4$ \\
\hline$p$ & \multicolumn{2}{|l|}{0.004} & \multicolumn{2}{|l|}{0.020} & \multicolumn{2}{|l|}{0.018} & \multicolumn{2}{|l|}{0.641} & \multicolumn{2}{|l|}{0.009} & \multicolumn{2}{|l|}{0.020} \\
\hline ETP & $1340 \pm 261$ & $1158 \pm 341$ & $1352 \pm 284$ & $1092 \pm 255$ & $1332 \pm 290$ & $1178 \pm 293$ & $1302 \pm 297$ & $1082 \pm 251$ & $1312 \pm 272$ & $808 \pm 355$ & $1382 \pm 258$ & $1080 \pm 277$ \\
\hline$p$ & \multicolumn{2}{|l|}{0.003} & \multicolumn{2}{|l|}{$<0.001$} & \multicolumn{2}{|l|}{0.008} & \multicolumn{2}{|l|}{0.011} & \multicolumn{2}{|l|}{0.001} & \multicolumn{2}{|l|}{\begin{tabular}{|l|}
0.001 \\
\end{tabular}} \\
\hline
\end{tabular}

Table 6. Association between clinical outcomes and baseline thrombin generation parameters obtained with tissue factor $(5 \mathrm{pM})$ in samples from hospitalized COVID-19 patients. Bold values denote statistical significance at the $p<0.05$ level. ARDS acute respiratory distress syndrome. ${ }^{*}$ Complications include ARDS, Thrombosis, Hemorrhage, or Death. $P$ values were based on Mann-Whitney-U test.

lytical data was performed. Peak values were dichotomized according to the median. As shown in Table 5, in COVID-19 patients higher LDH, CRP, IL-6, and D-dimer values were associated with longer lag-time triggered by TF. Additionally, increased peak LDH and D-dimer levels, but lower peak fibrinogen values were associated with lower ETP at 5 pM TF (Table 5).

Results from Spearman's correlation analysis for TGA parameters at $5 \mathrm{pM}$ TF, demonstrated associations with markers of cell lysis (LDH), inflammation (CRP, IL-6), and coagulation (fibrinogen and D-dimer): (1) the lag-time was positively associated with all the above biomarkers except for fibrinogen; (2) ETP was inversely associated with $\mathrm{LDH}$ and D-dimer, and positively related to fibrinogen levels, as shown in Table 5 and Supplementary Fig. S2.

Thrombin generation and clinical severity. We analyzed whether in COVID-19 patients, TGA measured in PPP with 5 pM TF associated with clinical complications. Outcomes of interest included CURB-65 score (a scoring system for assessing the severity of pneumonia), ICU admission, ARDS, death, thrombosis, and the composite for adverse events that includes ARDS, thrombosis, hemorrhage or death. Of the various outcomes considered, all of them showed a positive association with ETP, and also (except for death) with lagtime (Table 6).

As an additional main outcome, and according to the criteria defined by the ISTH to be applied to patients with a critical illness known to precipitate DIC, the ISTH-DIC score was evaluated, and the number of clinically relevant episodes (ADRS, thrombosis, bleeding or death) were also scored during follow-up. TGA showed lower ETP levels but longer lag-times in patients who experienced more clinical events and had higher ISTH-DIC scores (Fig. 1) (Table 6).

Predictive value of thrombin generation assay in COVID-19. Cox regression analysis was performed to evaluate the predictive value for baseline TGA parameters of adverse clinical events in patients hospitalized with COVID-19 infection. For this analysis, individuals experiencing adverse events within three days following sample collection were excluded $(\mathrm{N}=6)$. Cox regression analysis confirmed that in spite of the low number of events, baseline ETP and baseline D-dimer significantly predicted adverse outcomes (thrombosis, bleeding or death), being the baseline D-dimer/ETP ratio the best predictor (Fig. 2). 
A

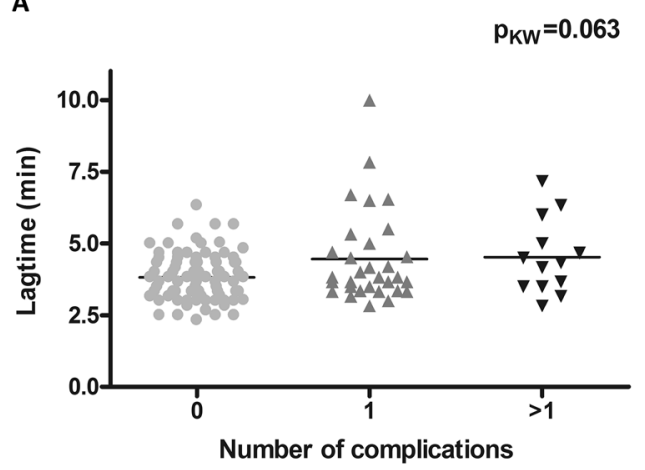

B

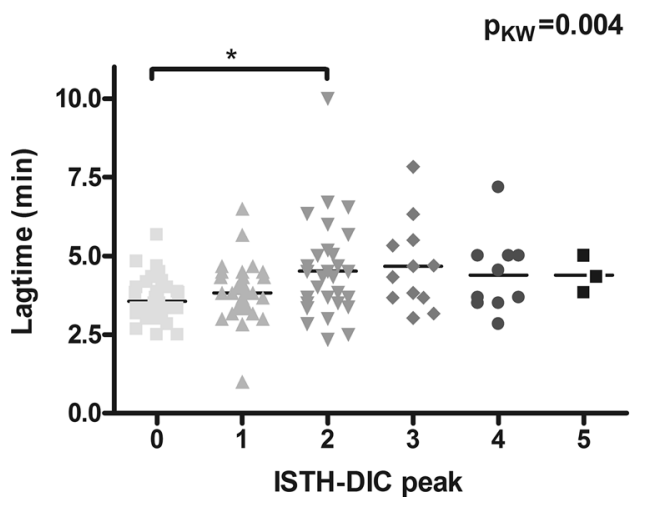

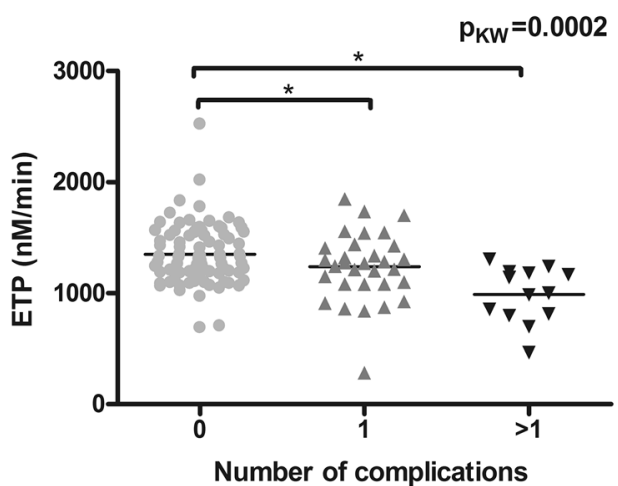

Number of complications

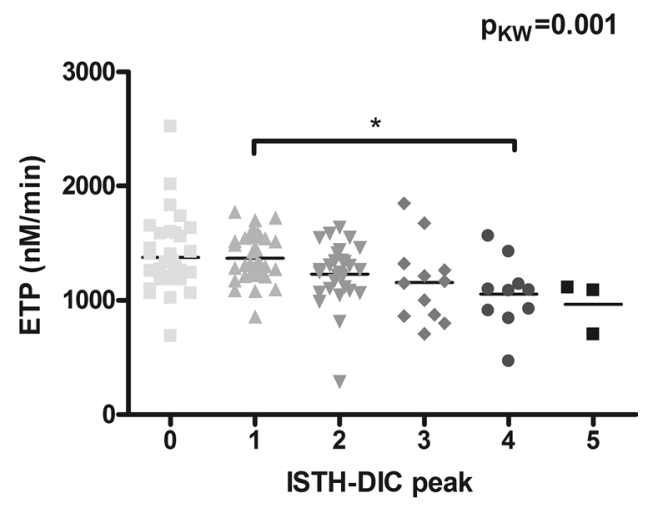

Figure 1. Association of lag-time and ETP values of COVID-19 patients with the number of complications (A) and peak ISTH-DIC scores (B). $\mathrm{p}_{\mathrm{KW}}$ : $p$ value based on Kruskal Wallis test comparing all groups; ${ }^{*} p<0.05$ based on Mann-Whitney-U test comparing pairs.

The AUC of the ROC curve of the baseline D-dimer/ETP ratio for adverse events in COVID-19 patients was 0.833 (95\%CI $0.719-0.948, p<0.001$ ) (Fig. 3A). The ROC curve showed that the cut-off value of 1.63 had a sensitivity of $83 \%$ and specificity of $75 \%$ for predicting adverse events. Using this optimal cut-off value, the Kaplan-Meier estimation also reflected that the baseline D-dimer/ETP ratio was significantly related to adverse events ( $p=0.016$, HR 0.153) (Fig. 3B).

\section{Discussion}

It is now accepted that coagulopathy and DIC associate with a poor prognosis in patients with COVID-19, although it is as yet unknown if this is directly related to SARS-CoV-2 damage, or more likely due to the effects of hypoxia and sepsis ${ }^{27}$. The high incidence of thrombosis in COVID-19 strongly supports a significant disturbance of the hemostatic system in these patients, that however is only mainly sustained by the increase of D-dimer ${ }^{28}$. Thus, further clues on the status of the hemostatic system in patients with COVID-19 are required. Moreover, as subsequent waves of infections appear, we need new and stronger prognostic biomarkers to guide clinical decision making in order to triage which patients can be discharged or may need admission, or to identify high-risk cases that might need admission in ICU. At the present time, current prophylaxis seems to be inefficient in some patients, which could however associate with an increased bleeding risk ${ }^{29}$, so that more individualized approaches to guide thrombosis prevention could help in the tailored management of patient ${ }^{30}$. Routine coagulation tests do not seem useful to discriminate severe cases of COVID-19, as recently suggested ${ }^{19}$. Clotting times (PT and aPTT) do not enable the correct appreciation of coagulation profile in patients with coagulopathy because they take into account only extreme reductions in the levels of pro-coagulant factors and are not sensitive to the concomitant reduction of inhibitors. In this framework, global tests of hemostasis, which are closer to the in vivo coagulation conditions and reflect the capacity of response of the hemostatic system to a triggering factor, might be of great value ${ }^{31}$. However, to our knowledge only three studies have used other global hemostatic tests apart from coagulation times to evaluate the hemostatic system in patients with COVID-19. Such studies, two using thromboelastography and the third also using a TGA, included a relatively limited number of patients $(22,24$ and 78 , respectively), and the first was restricted to patients admitted to ICU $\mathrm{U}^{32-34}$.

Here, we used a CAT TGA to analyze the response of the hemostatic system of baseline samples to TF both in the absence and presence of sTM (therefore evaluating the extrinsic pathway and the protein C system, respectively), but also by activating the contact pathway with silica. Additionally, we studied the association of baseline TGA parameters with analytical and clinical data, which may help to understand the relationship of changes in the hemostatic system with thromboinflammatory biomarkers and with clinical outcomes. For that, the assay 


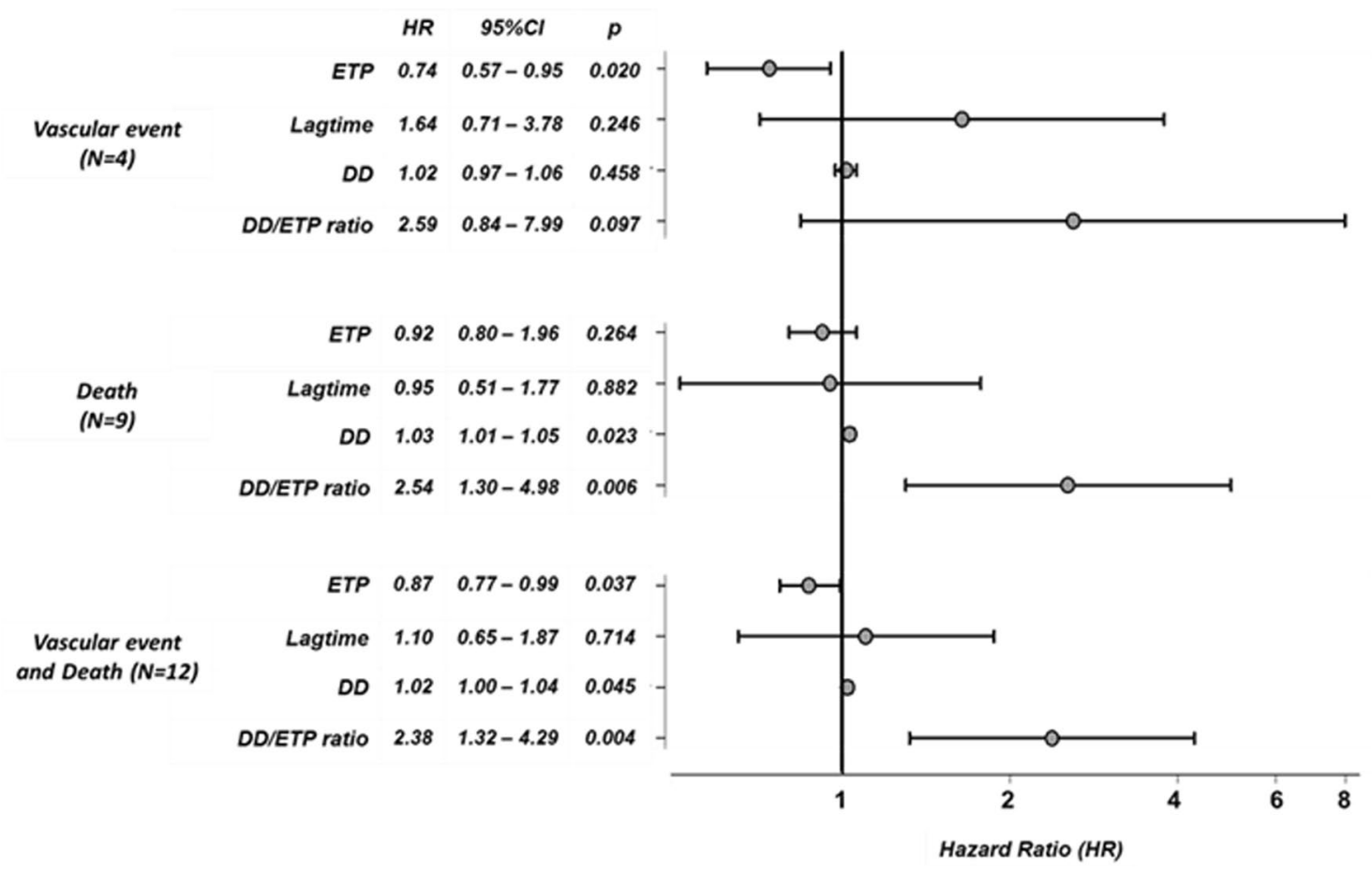

Figure 2. Cox regression analysis of baseline thrombin generation parameters, baseline D-dimer and the baseline D-dimer/ETP ratio in COVID-19 patients. Square root transformation of ETP and D-dimer and logarithmic transformation of D-dimer/ETP ratio were performed for predictive analysis. $95 \%$ confidence interval and $p$ value are shown for each Hazard Ratio. DD: D-dimer. HR Hazard ratio.

A

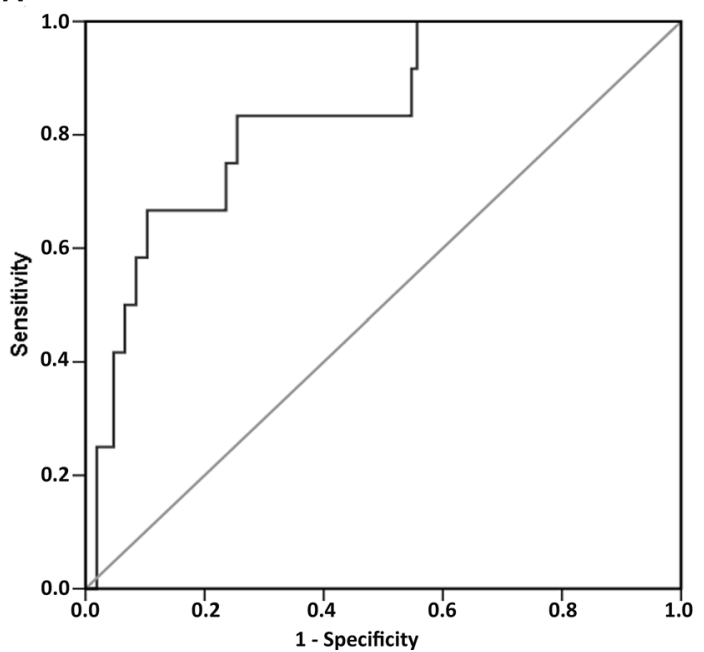

B

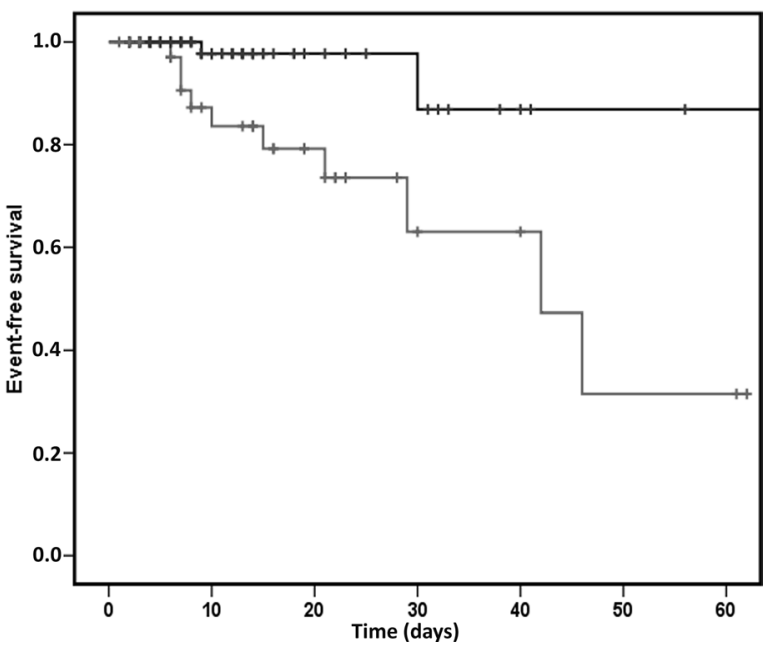

Figure 3. Ratio D-dimer/ETP as predictor for adverse events (vascular events or death) in COVID-19 patients. (A) ROC curve; (B) Kaplan-Meier curve.

was done in a single equipment under strict conditions aiming to reduce any variability, and with appropriate controls, which also included patients with SARS-CoV-2-negative pneumonia.

Results confirm previous findings showing a hypercoagulable state in COVID-19 patients, as they have similar thrombin generation than healthy controls despite thromboprophylaxis ${ }^{32-34}$. Results also reflect the impairment of the protein C pathway, as the addition of sTM, both in COVID-19, and in patients with SARS-CoV-2-negative pneumonia, resulted in minor reduction in the generation of thrombin compared to controls. These results are in line with those suggesting that infections may trigger the release of TM from the damaged endothelium ${ }^{35}$. This reduction of the antithrombotic capacity at the surface of the endothelium ${ }^{36,37}$, may contribute to fibrin deposition, particularly at the lung ${ }^{38}$. Further studies of sTM in these patients might be useful to validate this hypothesis. 


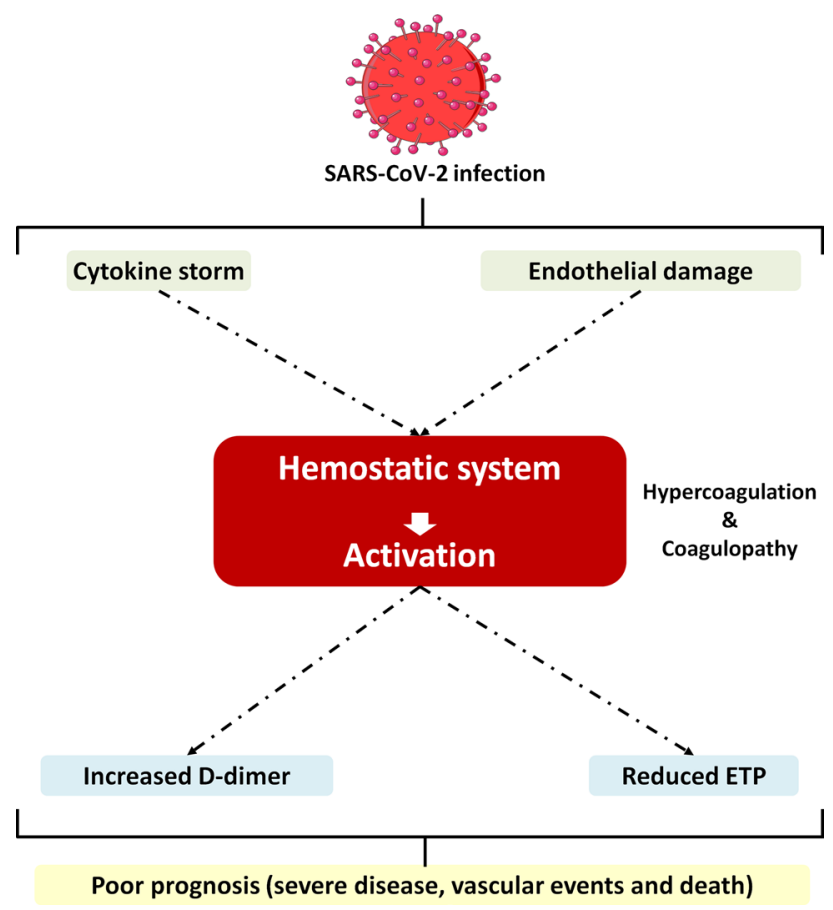

Figure 4. Schematic representation of potential insults associated to SARS-CoV-2 infection and the consequences on the hemostatic system. The drawing is a modified version of an image available in Servier Medical Art templates, which are licensed under a Creative Commons Attribution 3.0 Unported License; https:// smart.servier.com.

Our study also shows a good correlation of baseline TGA parameters with peak values of markers of cell lysis (LDH), inflammation (IL-6; CRP) and coagulation (fibrinogen and D-dimer). Interestingly, patients with higher inflammation and cell lysis have prolonged lag-time and " _reduced ETP, probably reflecting a consumption of coagulation factors of the hemostatic system. In this framework, the association of lag-time and ETP with $\mathrm{D}$-dimer is also remarkable. The fact that patients with higher $\mathrm{D}$-dimer had prolonged lag-time and reduced ETP supports a coagulopathy in severe COVID-19 patients. This finding was also reinforced by the excellent association of ETP with the ISTH-DIC score. Thus, our results would indicate both, a hypercoagulable state, and a low-grade coagulopathy in COVID-19 patients.

Finally, the association of baseline TGA parameters with clinical outcomes is also noteworthy. In contrast to that expected for a hypercoagulable state, lower ETP was associated to worse prognosis according to at-admission CURB-65 score and the development of adverse events (ARDS, vascular events, or death). Those patients with reduced capacity to generate thrombin have poor prognosis, suggesting that the severity of the disease is related to a low capacity of response of the hemostatic system, probably because of the associated consumptive coagulopathy. In accordance, our study confirms the prognostic value of D-dimer levels on mortality, which has been debated ${ }^{39}$. Additionally, these data also suggest that baseline TGA parameters, especially the baseline D-dimer/ETP ratio might have better prediction ability for vascular events and death. One of the strengths of the approach is the use of a representative patient sample that includes patients managed in two distinct institutions. But our study also has some limitations. First, we have no data about thrombin activity before initiation of heparin, which would allow more complete characterization of the effect of the anticoagulant on thrombin generation over time. Second, inherent to a retrospective analysis, there is a possibility that medical records were incomplete or missing data. Third, it would be interesting to evaluate whether factors such as age or sex, not matched between patients and controls in our study might play any role. Last, we did not perform a longitudinal follow-up analysis, and could not test dynamic changes of thrombin generation parameters. The variations over time of other coagulation measurements, such as prothrombin time activity ${ }^{40}$ and D-Dimer ${ }^{41}$, have already been shown to have prognostic value in patients hospitalized for COVID-19. Notwithstanding these limitations, our results suggest that thrombin generation could have a promising prognostic value in COVID-19 patients, but more extensive testing is warranted to validate these findings before being incorporated into clinical practice.

Overall, our study supports the results of previous reports showing multiple pathogenic pathways in COVID-19 patients. The severe "storm" of proinflammatory cytokines, combined with cell lysis, particularly at the endothelium, constitute insults leading to a significant hypercoagulable state that, despite antithrombotic prophylaxis, cause a consumptive and diffuse coagulopathy reflected by the increase of D-dimer, independently of the hypofibrinolysis that is also present in these patients ${ }^{42}$. Thus, patients with lower capacity of thrombin generation and higher D-dimer levels would have poor prognosis (Fig. 4). 


\section{Data availability}

The datasets used and/or analysed during the current study are available from the corresponding author on reasonable request.

Received: 30 October 2020; Accepted: 26 February 2021

Published online: 08 April 2021

\section{References}

1. Thomas, W. et al. Thrombotic complications of patients admitted to intensive care with COVID-19 at a teaching hospital in the United Kingdom. Thromb. Res. 191, 76-77 (2020).

2. Klok, F. A. et al. Confirmation of the high cumulative incidence of thrombotic complications in critically ill ICU patients with COVID-19: an updated analysis. Thromb. Res. 191, 148-150 (2020)

3. Llitjos, J. et al. High incidence of venous thromboembolic events in anticoagulated severe COVID-19 patients. J. Thromb. Haemost. 18, 1743-1746 (2020).

4. Helms, J. et al. High risk of thrombosis in patients with severe SARS-CoV-2 infection: a multicenter prospective cohort study. Intensive Care Med. 46, 1089-1098 (2020).

5. Poyiadji, N. et al. Acute Pulmonary embolism and COVID-19. Radiology 313, 129-131 (2020).

6. Jain, R. et al. COVID-19 related neuroimaging findings: a signal of thromboembolic complications and a strong prognostic marker of poor patient outcome. J. Neurol. Sci. 414, 116923 (2020).

7. Lodigiani, C. et al. Venous and arterial thromboembolic complications in COVID-19 patients admitted to an academic hospital in Milan Italy. Thromb. Res. 191, 9-14 (2020).

8. Hippensteel, J. A., Burnham, E. L. \& Jolley, S. E. Prevalence of venous thromboembolism in critically ill patients with COVID-19. Br. J. Haematol. 190, e134-e137 (2020).

9. Goshua, G. et al. Endotheliopathy in COVID-19-associated coagulopathy: evidence from a single-centre, cross-sectional study. Lancet. Haematol. 7, e575-e582 (2020).

10. Al-Ani, F., Chehade, S. \& Lazo-Langner, A. Thrombosis risk associated with COVID-19 infection: a scoping review. Thromb. Res. $192,152-160(2020)$

11. Cummings, M. J. et al. Epidemiology, clinical course, and outcomes of critically ill adults with COVID-19 in New York City: a prospective cohort study. Lancet (London, England) 395, 1763-1770 (2020).

12. Grasselli, G. et al. Risk factors associated with mortality among patients with COVID-19 in intensive care units in Lombardy Italy. JAMA Int. Med. 180, 1345 (2020).

13. Provazníková, D. et al. Seventeen novel SERPINC1 variants causing hereditary antithrombin deficiency in a Czech population. Thromb. Res. 189, 39-41 (2020).

14. Ranucci, M. et al. The procoagulant pattern of patients with COVID-19 acute respiratory distress syndrome. J. Thromb. Haemost. 18, 1747-1751 (2020).

15. Zhang, Y. et al. Profile of natural anticoagulant, coagulant factor and anti-phospholipid antibody in critically ill COVID-19 patients. J. Thromb. Thrombolysis 50, 580-586 (2020).

16. Iba, T. et al. The unique characteristics of COVID-19 coagulopathy. Crit. Care 24, 360 (2020).

17. Pons, S., Fodil, S., Azoulay, E. \& Zafrani, L. The vascular endothelium: the cornerstone of organ dysfunction in severe SARS-CoV-2 infection. Crit. Care 24, 353 (2020).

18. Dolhnikoff, M. et al. Pathological evidence of pulmonary thrombotic phenomena in severe COVID-19. J. Thromb. Haemost. 18, $1517-1519(2020)$

19. Iba, T., Levy, J. H., Levi, M., Connors, J. M. \& Thachil, J. Coagulopathy of Coronavirus Disease 2019. Crit. Care Med. 48, 1358-1364 (2020).

20. Colling, M. E. \& Kanthi, Y. COVID-19-associated coagulopathy: an exploration of mechanisms. Vasc. Med. https://doi.org/10. $1177 / 1358863 X 20932640(2020)$.

21. Whyte, C. S., Morrow, G. B., Mitchell, J. L., Chowdary, P. \& Mutch, N. J. Fibrinolytic abnormalities in acute respiratory distress syndrome (ARDS) and versatility of thrombolytic drugs to treat COVID-19. J. Thromb. Haemost. 18, 1548-1555 (2020).

22. World Health Organization. Clinical management of severe acute respiratory infection when novel coronavirus (nCoV) infection is suspected. Who (2020).

23. Toh, C. H. \& Hoots, W. K. The scoring system of the Scientific and Standardisation Committee on Disseminated Intravascular Coagulation of the International Society on Thrombosis and Haemostasis: a 5-year overview. J. Thromb. Haemost. 5, 604-606 (2007).

24. Hemker, H. C. et al. Calibrated automated thrombin generation measurement in clotting plasma. Pathophysiol. Haemost. Thromb. 33, 4-15 (2003).

25. Perrin, J. et al. Coagulation phenotypes in septic shock as evaluated by calibrated automated thrombography. Shock 43, 74-79 (2015).

26. StataCorp. Stata Statistical Software: Release 14. College Station, TX: StataCorp LP. 2015 (2015)

27. Tang, N., Li, D., Wang, X. \& Sun, Z. Abnormal coagulation parameters are associated with poor prognosis in patients with novel coronavirus pneumonia. J. Thromb. Haemost. 18, 844-847 (2020).

28. Al-Samkari, H. et al. COVID-19 and coagulation: bleeding and thrombotic manifestations of SARS-CoV-2 infection. Blood 136, 489-500 (2020).

29. Thachil, J. et al. ISTH DIC subcommittee communication on anticoagulation in COVID-19. J. Thromb. Haemost. 18, 2138-2144 (2020).

30. Pesavento, R. et al. The hazard of (sub)therapeutic doses of anticoagulants in non-critically ill patients with Covid-19: The Padua province experience. J. Thromb. Haemost. 18, 2629-2635 (2020).

31. Regnault, V., Hemker, H. C., Wahl, D. \& Lecompte, T. Phenotyping the haemostatic system by thrombography: potential for the estimation of thrombotic risk. Thromb. Res. 114, 539-545 (2004).

32. Spiezia, L. et al. COVID-19-related severe hypercoagulability in patients admitted to intensive care unit for acute respiratory failure. Thromb. Haemost. 120, 998-1000 (2020).

33. Panigada, M. et al. Hypercoagulability of COVID-19 patients in intensive care unit: a report of thromboelastography findings and other parameters of hemostasis. J. Thromb. Haemost. 18, 1738-1742 (2020).

34. Nougier, C. et al. Hypofibrinolytic state and high thrombin generation may play a major role in SARS-COV2 associated thrombosis. J. Thromb. Haemost. 18, 2215-2219 (2020).

35. Iba, T. \& Levy, J. H. Inflammation and thrombosis: roles of neutrophils, platelets and endothelial cells and their interactions in thrombus formation during sepsis. J. Thromb. Haemost. 16, 231-241 (2018).

36. Seigneur, M. et al. Plasma thrombomodulin: new approach of endothelium damage. Int. Angiol. 12, 355-359 (1993).

37. Remková, A., Kováčová, E., Príkazská, M. \& Kratochvílová, H. Thrombomodulin as a marker of endothelium damage in some clinical conditions. Eur. J. Intern. Med. 11, 79-84 (2000). 
38. Menter, T. et al. Postmortem examination of COVID-19 patients reveals diffuse alveolar damage with severe capillary congestion and variegated findings in lungs and other organs suggesting vascular dysfunction. Histopathology 77, 198-209 (2020).

39. Gris, J., Quéré, I., Pérez-Martin, A., Lefrant, J. \& Sotto, A. Uncertainties on the prognostic value of D-dimers in COVID-19 patients. J. Thromb. Haemost. 18, 2066-2067 (2020).

40. Luo, H., You, C., Lu, S. \& Fu, Y. Characteristics of coagulation alteration in patients with COVID-19. Ann. Hematol. 100, 45-52 (2021).

41. Valerio, L. et al. Course of D-dimer and C-reactive protein levels in survivors and nonsurvivors with COVID-19 pneumonia: a retrospective analysis of 577 patients. Thromb. Haemost. 121, 098-101 (2021).

42. Medcalf, R. L., Keragala, C. B. \& Myles, P. S. Fibrinolysis and COVID-19: a plasmin paradox. J. Thromb. Haemost. 18, 2118-2122 (2020).

\section{Acknowledgements}

We thank the participants involved in this study. We thank Dr. Alfonso Pérez (Clinical Analysis Laboratory Service, Hospital Morales Meseguer); Dr. Francisco Ortuño (Hematology and Clinical Oncology Service, Hospital Morales Meseguer) and Dr. Francisco Cañizares (Clinical Analysis Laboratory Service, Hospital Reina Sofia) for providing analytical data.

\section{Author contributions}

M.E.M-B., C.B-P., A.M. and B.M-B. recruited the samples and performed experimental analysis. M.E.M-B., C.B-P. and M.P.F-P. statistically analyzed all data. E.B., J.M.G-V., M.T.H. recruited patients and clinical outcomes and designed the research. V.V., J.C., M.L.L. designed the research, wrote the paper. All authors read and approved the final manuscript.

\section{Funding}

This work was supported by the 19873/GERM/15 and 00001/COVI/20 projects (Fundación Séneca). MEM-B holds a postdoctoral fellowship from Universidad de Murcia. CB-P holds the Río Hortega fellowship from Instituto de Salud Carlos III. BM-B holds a predoctoral fellowship from Fundación Séneca.

\section{Competing interests}

The authors declare no competing interests.

\section{Additional information}

Supplementary Information The online version contains supplementary material available at https://doi.org/ 10.1038/s41598-021-85906-y.

Correspondence and requests for materials should be addressed to J.C.

Reprints and permissions information is available at www.nature.com/reprints.

Publisher's note Springer Nature remains neutral with regard to jurisdictional claims in published maps and institutional affiliations.

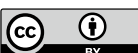

Open Access This article is licensed under a Creative Commons Attribution 4.0 International License, which permits use, sharing, adaptation, distribution and reproduction in any medium or format, as long as you give appropriate credit to the original author(s) and the source, provide a link to the Creative Commons licence, and indicate if changes were made. The images or other third party material in this article are included in the article's Creative Commons licence, unless indicated otherwise in a credit line to the material. If material is not included in the article's Creative Commons licence and your intended use is not permitted by statutory regulation or exceeds the permitted use, you will need to obtain permission directly from the copyright holder. To view a copy of this licence, visit http://creativecommons.org/licenses/by/4.0/.

(C) The Author(s) 2021 